\title{
Biological noise and H2A.Z: a promising connection for language
}

\author{
Antonio Benítez-Burraco * \\ Spanish Philology and its Didactics, University of Huelva, Huelva, Spain \\ *Correspondence: antonio.benitez@dfesp.uhu.es \\ Edited by: \\ Firas H. Kobeissy, University of Florida, USA \\ Reviewed by: \\ Bilal Fadlallah, Georgia Institute of Technology, USA \\ Chenggang Yu, Henry M. Jackson Foundation for the Advancement of Military Medicine, USA
}

Keywords: biological noise, canalization, language evolution, anatomically-modern humans, Neanderthals, H2A.Z, RUNX2, SIRT1

\section{A commentary on}

How does evolution tune biological noise? by Richard $M$ and Yvert $G$ (2014) Front. Genet. 5:374. doi: 10.3389/fgene.2014. 00374

In their paper "How does evolution tune biological noise?" (Front. Genet. 5:374) Richard and Yvert (2014) argue that biological noise (a source of variation regarding phenotypic traits) is under genetic control and consequently, is an evolvable trait. The authors focus on H2A.Z to illustrate their hypothesis and claim that this gene may act as a buffering device for this kind of variation. The examples provided by them range from $S$. cerevisae to $A$. thaliana, but we think that considerations from the fields of language disorders and language evolution may be adduced to lend credence to their hypothesis. This is the main goal of this commentary. Additionally, we will present evidence showing that H2A.Z is a gene of interest regarding the genetic underpinnings of the human faculty for language.

In a recent paper (Benítez-Burraco and Boeckx, 2014) we have claimed that, contrary to what has usually been assumed by linguists, the human faculty for language is not a homogeneous biological entity. Specifically, we showed that variation pervades language at all levels of analysis, from the neurobiological to the genetic layer. We further argued that this variation is canalized, to the extent that a functional language faculty almost invariably emerges in all subjects at the term of growth. What is more, environmental and endogenous perturbations (for example, in the form of mutations in genes related to language) bring about a limited set of deviant phenotypes only (i.e., language disorders), which can be conceived as decanalized states resulting from the uncovering of cryptic variation (following Gibson, 2009). In our paper we further claimed that, because of its biological novelty, the emergence of the language faculty can be conceived as a set of changes that decanalized the primate cognome, pushing it away from the robust equilibrium achieved after millions of stabilizing selection. Consequently, one would expect that some buffering mechanism have been reinforced in modern humans (henceforth AMHs) in order to recanalize all this new variation and make the faculty of language as robust as it is in current human populations.

In parallel to this line of argument, we have put forth in a recent series of papers (Boeckx and Benítez-Burraco, 2014a,b) a gene network that we think is implicated in the development of the language-ready brain, that is, our species-specific brain properties that enable us to acquire and use languages (see Arbib, 2012 for details). According to our view this languagereadiness resulted from a rewiring of the connections between sub-cortical (specifically, the thalamus) and cortical structures, a rewiring that is also manifested in a new developmental trajectory of skull formation, which gives rise to the characteristic globular head shape of AMHs, resulting from a relative expansion of the parietal and cerebellar regions compared to extant primates and extinct hominins (Neubauer et al., 2010). Our set of genes is centered on RUNX2, which controls different aspects of skull and brain development
(Stein et al., 2004; Reale et al., 2013) and whose promoter region shows two derived alleles in AMHs (Perdomo-Sabogal et al., 2014).

It is with this background in mind that we have approached Richard and Yvert's (2014) take on H2A.Z. One of the genes that control H2A.Z levels is SIRT1. SIRT1 reduces H2A.Z levels via proteasomemediated degradation (Baptista et al., 2013); not surprisingly given its role in the control of noise, as discussed by Richard and Yvert, high levels of H2A.Z (and low levels of SIRT1) are observed in different cancers (Baptista et al., 2013 and references herein). SIRT1 was known to be involved in cell survival, differentiation, and metabolism, but ample evidence now suggests that in the brain SIRT1 regulates different neural processes via deacetylation of histones and other proteins (like AKT1), including axon formation and elongation (Li et al., 2013), neural precursor activity and differentiation (e.g., high levels of SIRT1 signaling prevents neurogenesis and neural differentiation) (Saharan et al., 2013), and memory formation (Gao et al., 2010). Interestingly, H2A.Z has been recently linked to cognitive function too, as a direct regulator of memory consolidation systems (Zovkic et al., 2014). At the same time, a functional link also exists between SIRT1 and RUNX2. During osteogenesis SIRT1 increases the mRNA levels of RUNX2 and deacetylates RUNX2, ultimately promoting osteoblast differentiation (Shakibaei et al., 2012; Srivastava et al., 2012). The higher level of RUNX2 in the brain, the shorter interval time in which skull sutures remain open (Stein et al., 2004). Overall, 
a functional links seems to exist between SIRT1, RUNX2, and H2A.Z.

In our 2014a piece, we hypothesized that lower levels of RUNX2 in AMHs compared to Neanderthals (plausibly resulting from the changes in RUNX2 promoter) may explain some of the differences between the AMH and the Neanderthal skulls, brains, and cognitive abilities. Importantly, lower levels of RUNX2 are expected to correlate with lower levels of SIRT1 and with higher levels of H2A.Z at the brain level. If Richard and Yvert (2014) are right, this circumstance may have helped to buffer the molecular noise resulting from the changes occurred in the RUNX2 network that brought about a language-ready brain (and ultimately, from the decanalization of the primate cognome). But because of the involvement of both SIRT1 and H2A.Z in memory consolidation, we should expect that it contributed as well to the remodeling of the memory systems that is characteristic of our species, in particular, to the enhancement of the working memory and to a faster transition from declarative to procedural performance, a process important for language evolution and to which FOXP2, the famous language gene, also contributes to Schreiweis et al. (2014). Further evidence confirms the relevance of both genes for the emergence of modern language. Hence, SIRT1 is functionally related to some other genes encompassing the network we think important for the language-readiness, including TP53, EP300, HES1, and CLOCK (see Boeckx and Benítez-Burraco, 2014a,b for details). Similarly, according to String 9.1 [a tool that predicts direct/physical and indirect/functional associations between proteins based on genomic context, highthroughput experiments, conserved coexpression, and text mining (Szklarczyk et al., 2011)], some of the genes that have changed in AMHs compared to Neanderthals/Denisovans and that are part of our network are co-expressed along with H2A.Z, namely SPAG5 and ANAPC10 (see Benítez-Burraco and Boeckx, submitted for details).

In sum, we regard the hypothesis by Richard and Yvert of outstanding interest concerning language evolution and the biological nature of the human fac- ulty for language. Moreover, we think that the genetic aspects highlighted here, if explored experimentally, will allow us to gain a better view of the genetic underpinnings of language and the molecular mechanisms that channel variation at all levels of analysis.

\section{ACKNOWLEDGMENTS}

I would like to thank Cedric Boeckx for a fruitful collaboration over the years in the research on the biological underpinnings of language. Many of his thoughts and insights on this issue have contributed to improve this piece. The present work was made possible through a grant from the Spanish Ministry of Economy and Competitiveness (FFI-2013-43823-P).

\section{REFERENCES}

Arbib, M. (2012). How the Brain Got Language. Oxford: Oxford University Press.

Baptista, T., Graça, I., Sousa, E. J., Oliveira, A. I., Costa, N. R., Costa-Pinheiro, P., et al. (2013). Regulation of histone H2A.Z expression is mediated by sirtuin 1 in prostate cancer. Oncotarget 4, 1673-1685.

Benítez-Burraco, A., and Boeckx, C. (2014). Universal Grammar and biological variation: an EvoDevo agenda for comparative biolinguistics. Biol. Theor. 9, 122-134. doi: 10.1007/s13752-014-0164-0

Boeckx, C., and Benítez-Burraco, A. (2014a). The shape of the human language-ready brain. Front. Psychol. 5:282. doi: 10.3389/fpsyg.2014.00282

Boeckx, C., and Benítez-Burraco, A. (2014b). Globularity and language-readiness: Generating new predictions by expanding the set of genes of interest. Front. Psychol. 5:1324. doi: 10.3389/fpsyg.2014.01324

Gao, J., Wang, W. Y., Mao, Y. W., Gräff, J., Guan, J. S., Pan, L., et al. (2010). A novel pathway regulates memory and plasticity via SIRT1 and miR-134. Nature 466, 1105-1109. doi: 10.1038/nature09271

Gibson, G. (2009). Decanalization and the origin of complex disease. Nat. Rev. Genet. 10, 134-140. doi: $10.1038 / \mathrm{nrg} 2502$

Li, X. H., Chen, C., Tu, Y., Sun, H. T., Zhao, M. L., Cheng, S. X., et al. (2013). Sirt1 promotes axonogenesis by deacetylation of Akt and inactivation of GSK3. Mol. Neurobiol. 48, 490-499. doi: 10.1007/s12035-013-8437-3

Neubauer, S., Gunz, P., and Hublin, J.-J. (2010). Endocranial shape changes during growth in chimpanzees and humans: a morphometric analysis of unique and shared aspects. J. Hum. Evol. 59, 555-566. doi: 10.1016/j.jhevol.2010.06.011

Perdomo-Sabogal, A., Kanton, S., Walter, M. B., and Nowick, K. (2014). The role of gene regulatory factors in the evolutionary history of humans. Curr. Opin. Genet. Dev. 29C, 60-67. doi: 10.1016/j.gde.2014.08.007

Reale, M. E., Webb, I. C., Wang, X., Baltazar, R. M., Coolen, L. M., and Lehman, M. N. (2013). The transcription factor Runx 2 is under circadian control in the suprachiasmatic nucleus and functions in the control of rhythmic behavior. PLOS ONE 8:e54317. doi: 10.1371/journal.pone.0054317

Richard, M., and Yvert, G. (2014). How does evolution tune biological noise? Front. Genet. 5:374. doi: 10.3389/fgene.2014.00374

Saharan, S., Jhaveri, D. J., and Bartlett, P. F. (2013). SIRT1 regulates the neurogenic potential of neural precursors in the adult subventricular zone and hippocampus. J. Neurosci. Res. 91, 642-659. doi: 10.1002/jnr.23199

Schreiweis, C., Bornschein, U., Burguière, E., Kerimoglu, C., Schreiter, S., Dannemann, M., et al. (2014). Humanized Foxp2 accelerates learning by enhancing transitions from declarative to procedural performance. Proc. Natl. Acad. Sci. U.S.A. 111, 14253-14258. doi: 10.1073/pnas. 1414542111

Shakibaei, M., Shayan, P., Busch, F., Aldinger, C., Buhrmann, C., Lueders, C., et al. (2012). Resveratrol mediated modulation of Sirt-1/Runx2 promotes osteogenic differentiation of mesenchymal stem cells: potential role of Runx2 deacetylation. PLoS ONE 7:e35712. doi: 10.1371/journal.pone.0035712

Srivastava, S., Bedi, U., and Roy, P. (2012). Synergistic actions of insulin-sensitive and Sirt1-mediated pathways in the differentiation of mouse embryonic stem cells to osteoblast. Mol. Cell. Endocrinol. 361, 153-164. doi: 10.1016/j.mce.2012.04.002

Stein, G. S., Lian, J. B., van Wijnen, A. J., Stein, J. L., Montecino, M., Javed, A., et al. (2004). Runx2 control of organization assembly and activity of the regulatory machinery for skeletal gene expression. Oncogene 23, 4315-4329. doi: 10.1038/sj.onc. 1207676

Szklarczyk, D., Franceschini, A., Kuhn, M., Simonovic, M., Roth, A., Mínguez, P., et al. (2011). The STRING database in 2011: functional interaction networks of proteins, globally integrated and scored. Nucleic Acids Res. 39, D561-D568. doi: 10.1093/nar/gkq973

Zovkic, I. B., Paulukaitis, B. S., Day, J. J., Etikala, D. M., and Sweatt, J. D. (2014). Histone H2A.Z subunit exchange controls consolidation of recent and remote memory. Nature 515, 582-586. doi: $10.1038 /$ nature 13707

Conflict of Interest Statement: The author declares that the research was conducted in the absence of any commercial or financial relationships that could be construed as a potential conflict of interest.

Received: 24 November 2014; accepted: 16 December 2014; published online: 09 January 2015.

Citation: Benitez-Burraco A (2015) Biological noise and H2A.Z: a promising connection for language. Front. Genet. 5:463. doi: 10.3389/fgene.2014.00463

This article was submitted to Systems Biology, a section of the journal Frontiers in Genetics.

Copyright (C) 2015 Benitez-Burraco. This is an openaccess article distributed under the terms of the Creative Commons Attribution License (CC BY). The use, distribution or reproduction in other forums is permitted, provided the original author(s) or licensor are credited and that the original publication in this journal is cited, in accordance with accepted academic practice. No use, distribution or reproduction is permitted which does not comply with these terms. 